\title{
Supplement to "A Brief Tutorial on Transformation Based Markov Chain Monte Carlo and Optimal Scaling of the Additive Transformation"
}

\author{
Kushal Kr. Dey ${ }^{\dagger}$, Sourabh Bhattacharya ${ }^{\ddagger+}$ \\ $\dagger$ Department of Statistics, University of Chicago, USA \\ $\ddagger$ Interdisciplinary Statistical Research Unit, Indian Statistical Institute, Kolkata \\ + Corresponding author: bhsourabh@gmail.com
}

Throughout, we refer to our main manuscript Dey and Bhattacharya (2016) as DB.

\section{S-1 Computational efficiency of TMCMC}

It may seem that TMCMC is computationally more expensive because we are randomly generating $d+1$ many values $\left(\epsilon, b_{1}, b_{2}, \ldots, b_{d}\right)$ whereas in RWM, we are generating $d$ many random variables $\left(\epsilon_{1}, \epsilon_{2}, \ldots, \epsilon_{d}\right)$ where $\epsilon \sim N\left(0, \frac{\ell^{2}}{d}\right) I_{\{\epsilon>0\}}$ and $\epsilon_{i} \sim N\left(0, \frac{\ell^{2}}{d}\right) \forall i=1,2, \ldots, d$. However generating $b_{i}$ is equivalent to simple tosses of a fair coin which is a much easier exercise compared to drawing a set of independent normal random variables required by RWM. As a vindication of this, we obtain the average computation time (in seconds) of 100,00,00 iterations with RWM and TMCMC algorithms across dimensions 2 to 100, averaged over 100 replications of 100,00,00 iterations for each dimension, when the $d$-dimensional target density is a product of $d$ standard normals. The codes are written in $\mathrm{C}$ and implemented in an ordinary 32-bit laptop. The results of our experiment are displayed in Figure S-1. Fgure S-2 displays the computation times of RWM and TMCMC with respect to a single replication of the 100,00,00 iterations. In both the diagrams, TMCMC is seen to take significantly less computational time compared to the RWM algorithm, particularly in higher dimensions. Much longer runs, particularly in very high dimensions, would see TMCMC saving very substantial amount of computational time in comparison with RWM. For further discussion on computational gains of TMCMC over RWM, see Section 9 of DB.

It must be emphasized that the proposal density for $\epsilon$ in TMCMC can be any distribution on the positive support. Similarly, the RWM algorithm also does not require the proposal to be normal. However, the optimal scaling results for RWM inherently assume normality and for the sake of comparison, we have also restricted our focus on $\epsilon \sim N\left(0, \frac{\ell^{2}}{d}\right) I_{\{\epsilon>0\}}$ in the subsequent sections.

\section{S-2 Details on the need for optimal scaling of additive TMCMC}

We first try to impress the fact that too small or too large values of $\ell$ can both lead to poor performance of the chain and it is this trade-off that draws our interest in finding an optimal value of $\ell$. If the value of $\ell$ or equivalently, the proposal variance, is large, then the probability of a move falling in low density regions (with respect to the target density) of the space increases as the moves $\left(x_{1}+b_{1} \epsilon, \ldots, x_{d}+b_{d} \epsilon\right)$ are likely to be quite far apart from $\left(x_{1}, \ldots, x_{d}\right)$. This leads to smaller values of the ratio $\frac{\pi\left(x_{1}+b_{1} \epsilon, \ldots, x_{d}+b_{d} \epsilon\right)}{\pi\left(x_{1}, \ldots, x_{d}\right)}$ and thus lower acceptance rates. In fact, for high dimensions, this acceptance rate can be quite low for even moderately large values of 


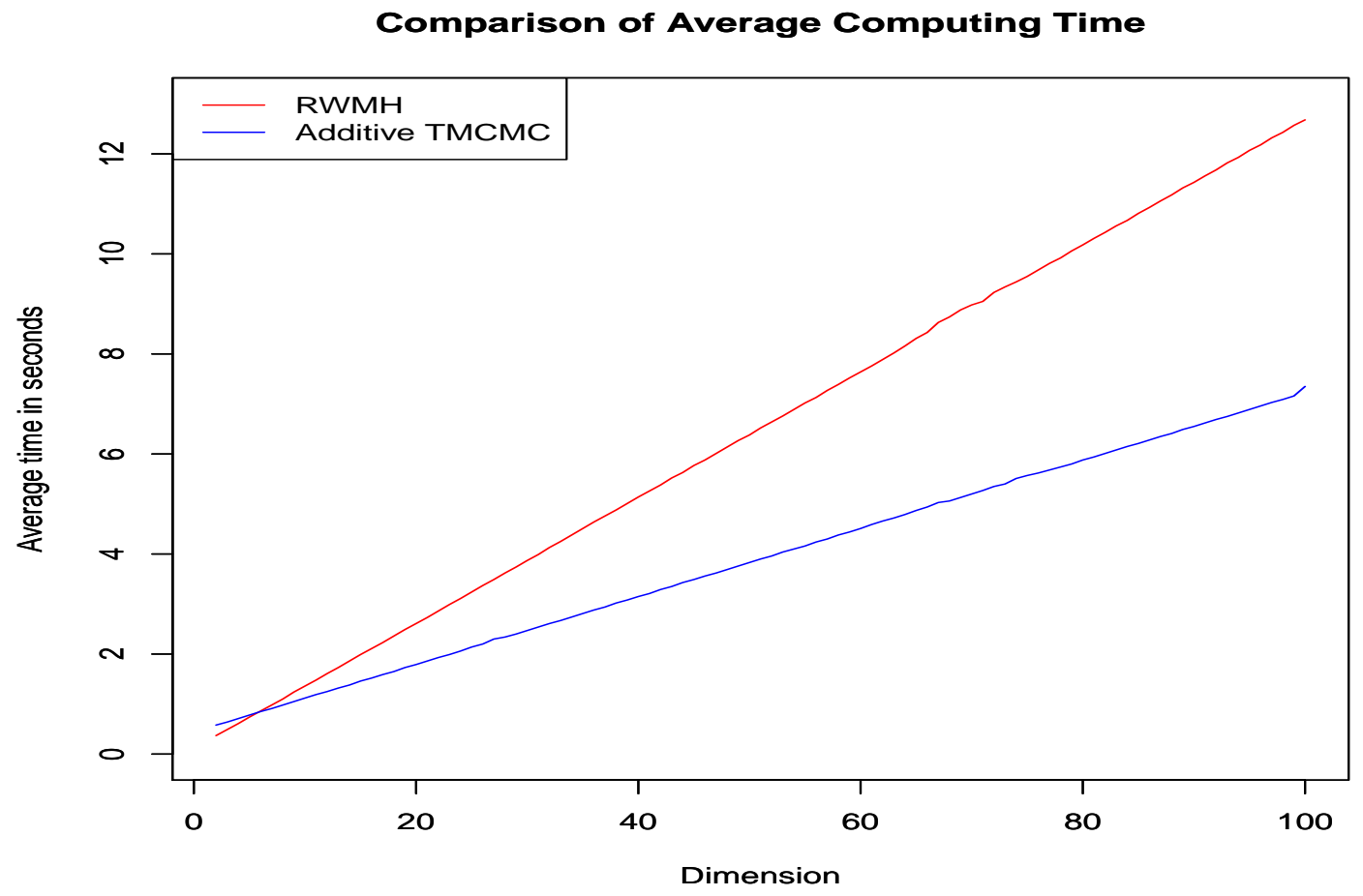

Figure S-1: Average computation time (in seconds) of 100,00,00 iterations with RWM and TMCMC algorithms corresponding to dimensions varying from 2 to 100 . TMCMC takes significantly less computation time compared to RWM, particularly in higher dimensions. The codes are written in $\mathrm{C}$ and implemented on an ordinary 32-bit laptop.

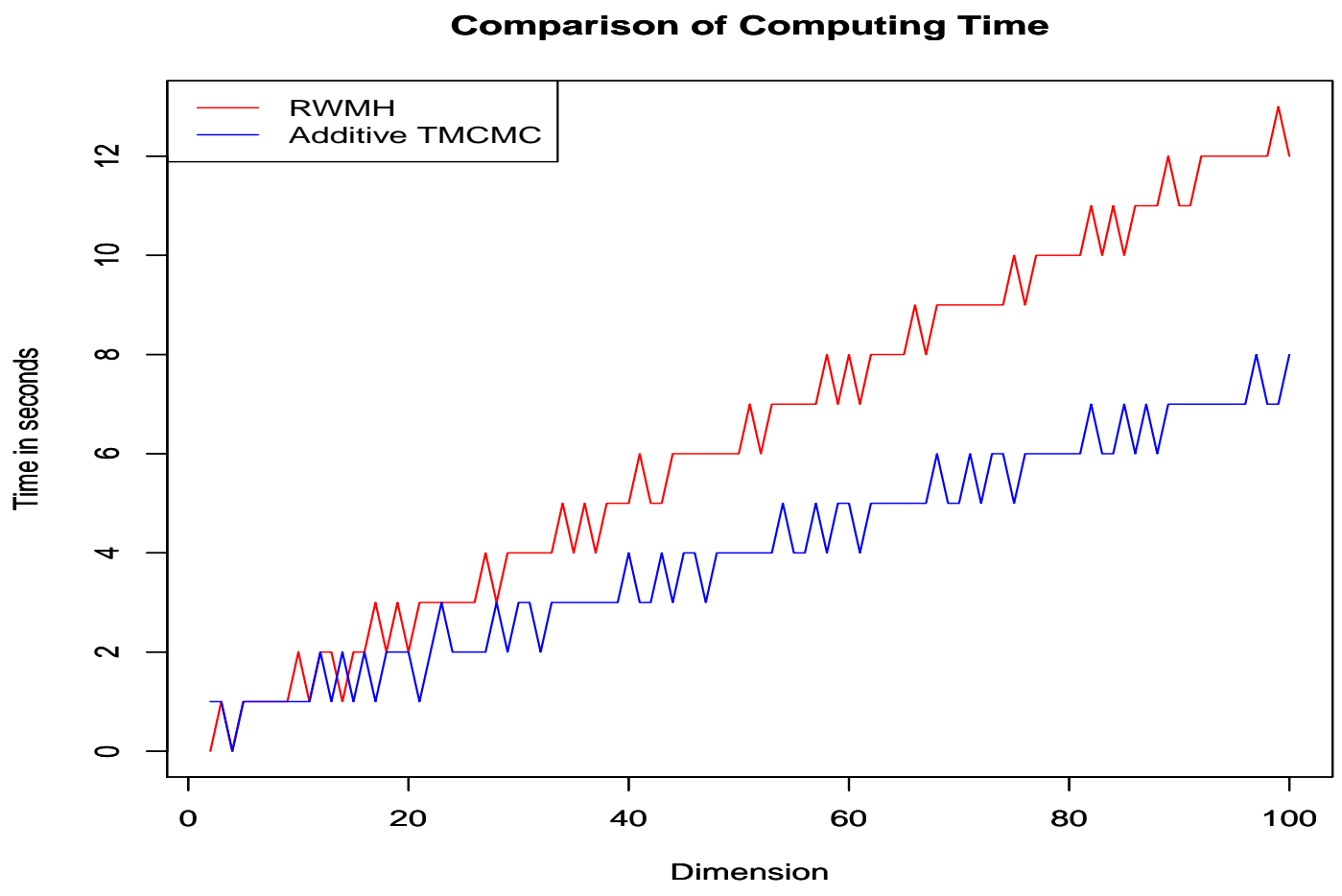

Figure S-2: Computation time (in seconds) of 100,00,00 iterations with RWM and TMCMC algorithms corresponding to dimensions varying from 2 to 100. TMCMC takes significantly less computation time compared to RWM, particularly in higher dimensions. The codes are written in C and implemented on an ordinary 32-bit laptop. 


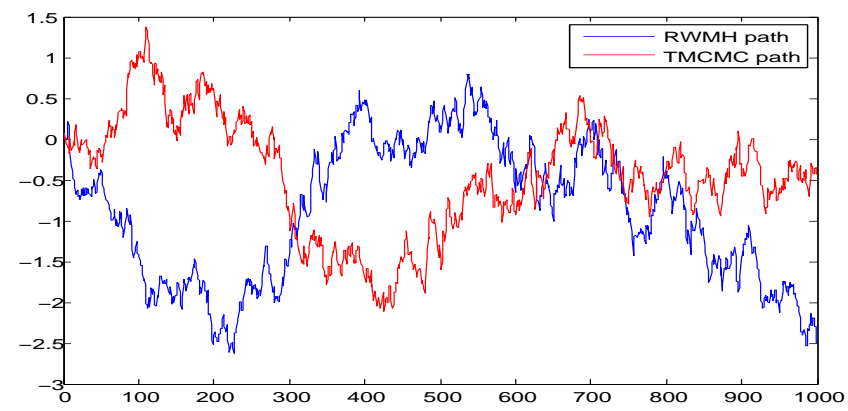

(a) Sample paths of RWM and additive TMCMC for small proposal variance.

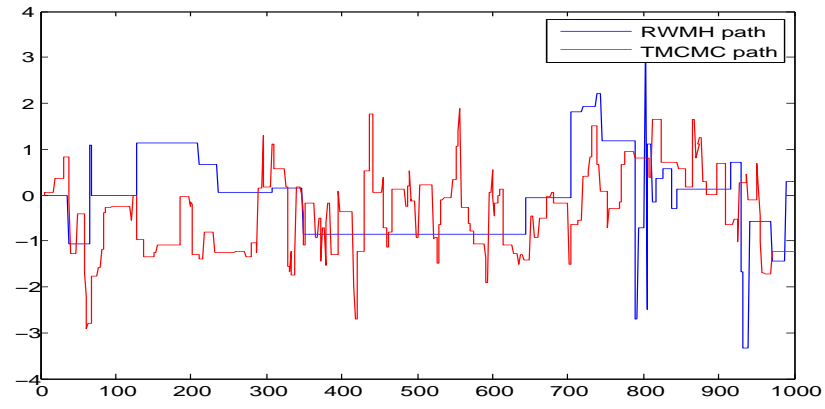

(b) Sample paths of RWM and additive TMCMC for large proposal variance.

Figure S-3: Comparison of RWM and additive TMCMC sample paths for small and large values of the proposal variance. The target density is $N(0,1)$, the standard normal distribution.

$\ell$. On the other hand, if the value of $\ell$ is too small, then the acceptance rate will be higher but we then have to compromise in terms of exploration of the space. Much of our moves will lie very close to the initial point and as a result, the chain will move very slowly. An instance of the movement of the RWM and additive TMCMC chain for significantly small and large values of $\ell$ are depicted in Figure S-3 assuming that the target distribution is standard normal. For small values of $\ell$, the fact that the chain moves slowly gets reflected in the autocorrelation factor (ACF) of the chain, which would be on the higher side (Figure S-4). All these motivate us to find an optimal value of $\ell$ that would take care of these problems. Our approach would be to derive the diffusion process approximation of the additive TMCMC process in the limit as $d \rightarrow \infty$ and then we maximize the diffusion speed or the rate of change of variance of the chain in the limit. Intuitively, if the scale factor $\ell$ is small, then starting from a point $X_{t}$ at time $t$, the moves corresponding to adjacent time points $X_{t+h}$ are quite close and so the limiting change of variation is quite small for the corresponding diffusion process. If on the other hand the scale factor $\ell$ is large, the chain hardly moves, and hence $X_{t+h}$ for sufficiently small $h$ are often same as $X_{t}$, thereby leading to lower value of diffusion speed. On optimizing the diffusion speed for the TMCMC chain, we obtain the optimal value of the acceptance rate to be 0.439 . Panels (a), (b) and (c) of Figure S-5 depict the path of the TMCMC chain for various choices of proposal variance, ranging from too small through the optimal value to quite large. Note that the target density is best approximated by the chain at optimal scaling. A better understanding of this is achieved by perceiving how well the histogram of observations obtained after running a chain up to a certain length of time, approximates the true density (panels (c), (d), (e) of Figure S-5). 

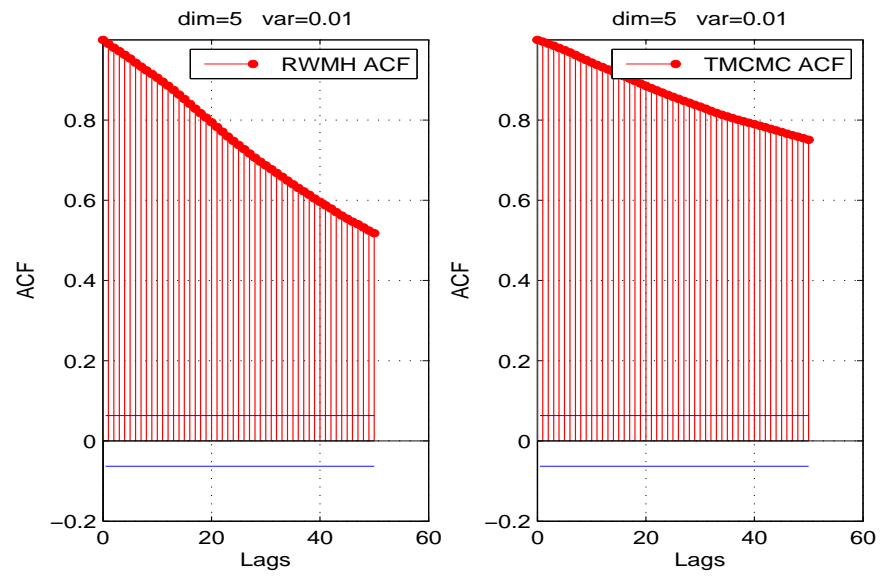

Figure S-4: ACFs of RWM and additive TMCMC for small proposal variance. The target density is $N(0,1)$, the standard normal distribution.

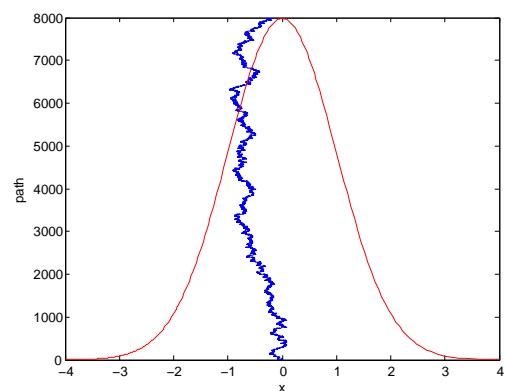

(a) $\ell=0.01$

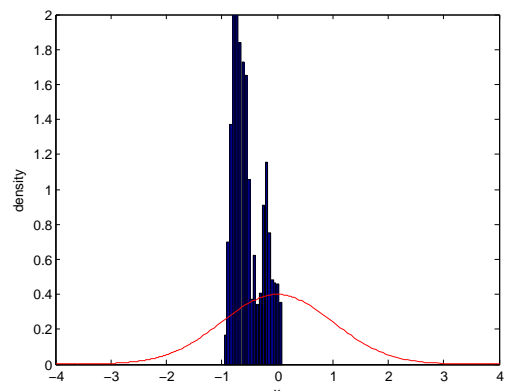

(d) $\ell=0.01$

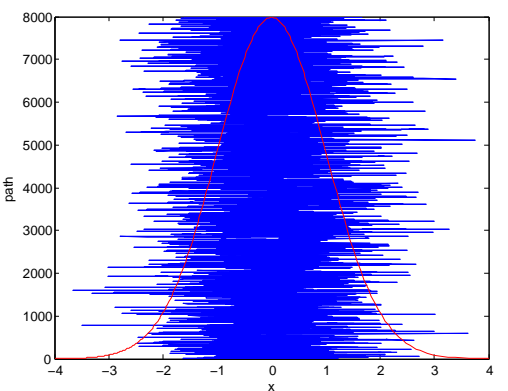

(b) $\ell=2.4$ (optimal)

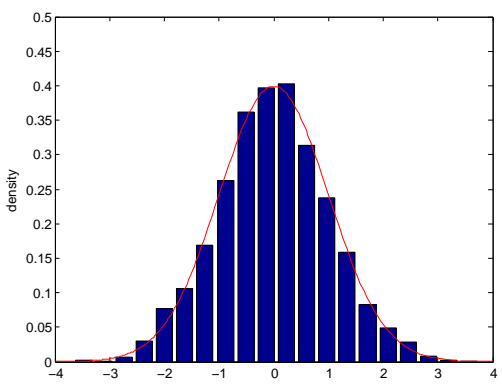

(e) $\ell=2.4$ (optimal)

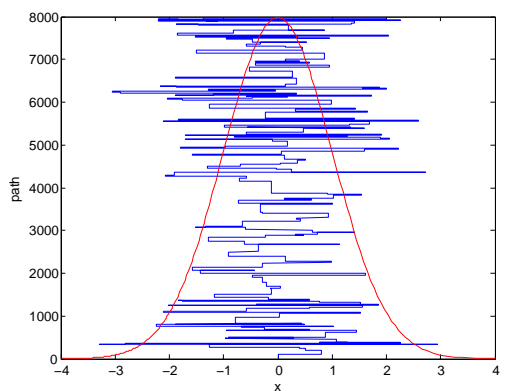

(c) $\ell=20$

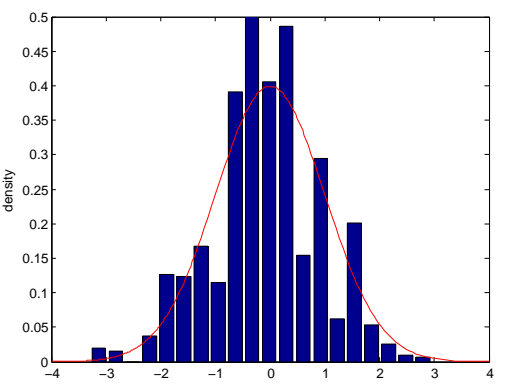

(f) $\ell=20$

Figure S-5: The upper panels (a), (b) and (c) show the paths of TMCMC chain for three various choices of scalings together with the target density $N(0,1)$ plot. These highlight how well the paths explore the given target density. The lower panels (c), (d) and (e) display the histograms of the sample observations obtained from TMCMC for these choices of scalings together with the target density $N(0,1)$. These highlight how well the histograms approximate the target density for the given run of TMCMC. 


\section{S-3 Discussion on consequences of non-robustness of RWM with respect to scale choices}

For general, $d$-dimensional target distributions, RWM entails the proposal with transitions of the form $\left(x_{1}, \ldots, x_{d}\right) \rightarrow\left(x_{1}+\frac{\ell_{1}}{\sqrt{d}} \epsilon_{1}, \ldots, x_{1}+\frac{\ell_{d}}{\sqrt{d}} \epsilon_{d}\right)$, where, for $i=1, \ldots, d, \epsilon_{i} \sim N(0,1)$, and $\ell_{i}$ are constants to be chosen appropriately. Often $\ell_{i}$ may be of the form $\ell a_{i}$, where $a_{i}$ may be needed to determine appropriately in addition to $\ell$. As instance of this form occurs in the dependent set-up of Mattingly et al. (2011), but $a_{i}$ in that set-up are the square roots of the eigenvalues of the Gaussian measure with independent components that is assumed to dominate the target density. However, in practice such assumption of independent Gaussian components will generally not hold, and hence it is far from straightforward to determine $a_{i}$ appropriately in realistic cases.

Since all the set-ups considered so far yield the optimal acceptance rate 0.234 for RWM, it may be anticipated that the result holds quite generally, and applied MCMC practitioners may be advised to tune $\left(\ell_{1}, \ldots, \ell_{d}\right)$ such that the acceptance rate is close to 0.234 . In fact, using a measure of efficiency which is the reciprocal of integrated autocorrelation time, Roberts and Rosenthal (2001) demonstrate that the RWM proposal may be tuned to achieve an acceptance rate between 0.15 to 0.5 , which would make the algorithm around $80 \%$ efficient. However, for large dimension $d$, appropriate tuning of so many scale parameters seems to be an extremely arduous task. In our simulations presented in Section 8 of DB we observe that even in the simple situation where the target density is an iid product of normal densities, when the dimension increases, particularly when $d=100$ and $d=200$, departure from the optimal scale results in drastic fall in acceptance rates, far below what is prescribed by Roberts and Rosenthal (2001); see Table 1 of DB. The diffusion speeds under such mis-specifications tend to be quite low because of non-robustness with respect to scale choice (see Figures $1-6$ of DB). Since low diffusion speed is equivalent to high autocorrelation (see equation (18) of Roberts and Rosenthal (2001)), the efficiency measures of Roberts and Rosenthal (2001) that use integrated autocorrelation, are also expected to indicate less efficiency. Thus, in summary manually tuning the RWM proposals appropriately in more general and complicated situations and in high dimensions seems to be a very daunting task. There are methods for automatically and adaptively tuning the scales to progress towards the desired optimal acceptance rate as number of iterations increases, but as we discuss in Section S-5 computationally this can be a very costly exercise in high dimensions.

\section{S-4 Discussion on possible advantages of additive TMCMC for relatively more robust behaviour with respect to scale choices}

Our results on optimal scaling offers the following general thumb rule to the users of additive TMCMC: tune the additive TMCMC proposal to achieve approximately $44 \%$ acceptance rate. Note that even though the optimal acceptance rate of additive TMCMC is significantly higher than that of RWM, both the algorithms have approximately the same optimal scalings that maximize the diffusion speeds (see Figures $1-6$ ).

The results of our simulation studies reported in Table 1 of DB demonstrate that even in dimension as low as $d=2$, our optimal acceptance rate 0.439 is remarkably accurate. The table further demonstrates that even if the scale of additive TMCMC is sub-optimally chosen, the acceptance rates remain higher than $20 \%$ for all dimensions, whereas for the same sub-optimal scale choice the acceptance rate of RWM falls to about $0.33 \%$ in high dimensions. Figures $1-6$ show that around the optimal scale, the diffusion speeds of additive TMCMC under various setups do not change significantly. Using the relationship between diffusion speed and the measure of efficiency proposed by Roberts and Rosenthal (2001) one can conclude that unlike RWM, 
the efficiency of additive TMCMC is not substantially affected by sub-optimal scale choices. Hence, tuning the additive TMCMC proposal is a far more safe and easy exercise compared to that of RWM. It seems to us that this is quite an advantage of additive TMCMC over RWM in general, high-dimensional set-ups. As we discuss in Section S-5 adaptive methods can be employed to approach the exact optimal acceptance rate 0.439 in substantially less number of iterations compared to adaptive RWM, facilitating huge computational gain over the latter.

\section{S-5 Discussion on adaptive versions of RWM and additive TM- CMC for enforcing optimal acceptance rates in complex, high-dimensional problems}

Adaptive MCMC methods (see, for example, Roberts and Rosenthal (2009) and the references therein) are designed to combat the difficulty of determining appropriate proposal scalings. In the context of RWM, various adaptive strategies are presented in Roberts and Rosenthal (2009) to choose the scalings in an adaptive manner so that the optimal acceptance rate 0.234 is achieved in the long run. Dey and Bhattacharya (2015) adopted the strategies in the case of additive TMCMC and made a detailed comparison with the corresponding adaptive RWM methods. In particular, they found that even after a very large number of iterations most of the the adaptive methods related to RWM yielded acceptance rates which are significantly different from 0.234 , while the adaptive TMCMC algorithms very quickly yielded acceptance rates reasonably close 0.439 , even in dimensions as low as $d=2$. This implies quite substantial savings of TMCMC in terms of computation time in comparison with RWM. Performance wise as well, the results of Dey and Bhattacharya (2015) favour adaptive TMCMC over adaptive RWM in high dimensions, with respect to the various measures which we also employ in this current work.

\section{S-6 Proofs of optimal scaling results of additive TMCMC}

\section{S-6.1 Proof of Theorem 3.1 of DB}

Proof. For our purpose, we define the discrete time generator of the TMCMC approach, as

$$
\begin{aligned}
G_{d} V(x)=\frac{d}{2^{d}} \sum_{\left\{\begin{array}{c}
b_{i} \in\{-1,+1\} \\
\forall i=1, \ldots, d
\end{array}\right\}} \int_{0}^{\infty}\left[\left(V\left(x_{1}+b_{1} \epsilon, \ldots, x_{d}+b_{d} \epsilon\right)-V\left(x_{1}, \ldots, x_{d}\right)\right)\right. \\
\left.\times\left(\min \left\{1, \frac{\pi\left(x_{1}+b_{1} \epsilon, \ldots, x_{d}+b_{d} \epsilon\right)}{\pi\left(x_{1}, x_{2}, \ldots, x_{d}\right)}\right\}\right)\right] q(\epsilon) d \epsilon .
\end{aligned}
$$

Since by our assumption $(\log f)^{\prime}$ is Lipschitz, in the above equation we may assume that $V$ belongs to the space of inifinitely differentiable functions with compact support (see, for example, Bedard (2007) for further details).

The Skorohod topology allows us to treat $G_{d}$ as a continuous time generator that has jumps at the rate $d^{-1}$. Given our restricted focus on a one dimensional component of the actual process, we assume $V$ to be a function of the first co-ordinate only. Under this assumption, the generator defined in (1) is a function of only $\epsilon$ and $b_{1}$, and can be rephrased as 


$$
\begin{aligned}
G_{d} V(x)=\frac{d}{2} \int_{0}^{\infty} \sum_{b_{1} \in\{-1,+1\}}[ & \left(V\left(x_{1}+b_{1} \epsilon\right)-V\left(x_{1}\right)\right) \\
& \left.\times E_{b_{2}, \ldots, b_{d}}\left(\min \left\{1, \frac{\pi\left(x_{1}+b_{1} \epsilon, \ldots, x_{d}+b_{d} \epsilon\right)}{\pi\left(x_{1}, \ldots, x_{d}\right)}\right\}\right)\right] q(\epsilon) d \epsilon,
\end{aligned}
$$

where $E_{b_{2}, \ldots, b_{d}}$ is the expectation taken conditional on $b_{1}$ and $\epsilon$.

First we show that the quantity $G_{d} V(x)$ is a bounded quantity.

$$
\begin{aligned}
G_{d} V(x) & \leq d E_{\left\{b_{1}, \epsilon\right\}}\left[V\left(x_{1}+b_{1} \epsilon\right)-V\left(x_{1}\right)\right] \\
& =d V^{\prime}\left(x_{1}\right) E_{\left\{b_{1}, \epsilon\right\}}\left(b_{1} \epsilon\right)+\frac{d}{2} V^{\prime \prime}\left(x_{1}^{*}\right) E_{\left\{b_{1}, \epsilon\right\}}\left(\epsilon^{2}\right) \\
& \leq \ell^{2} K
\end{aligned}
$$

where $x_{1}^{*}$ lies between $x_{1}$ and $x_{1}+b_{1} \epsilon$ and $K$ is the maximum value of $V^{\prime \prime}$.

Note that

$$
\begin{aligned}
E_{b_{2}, \ldots, b_{d}} & {\left[\min \left\{1, \frac{\pi\left(x_{1}+b_{1} \epsilon, \ldots, x_{d}+b_{d} \epsilon\right)}{\pi\left(x_{1}, \ldots, x_{d}\right)}\right\}\right] } \\
=\quad & E_{b_{2}, \ldots, b_{d}}\left[\operatorname { m i n } \left\{1, \exp \left(\log \left(f\left(x_{1}+b_{1} \epsilon\right)\right)-\log \left(f\left(x_{1}\right)\right)\right.\right.\right. \\
& \left.\left.+\sum_{j=2}^{d}\left\{b_{j} \epsilon\left\{\log \left(f\left(x_{j}\right)\right)\right\}^{\prime}+\frac{\epsilon^{2}}{2 !}\left\{\log \left(f\left(x_{j}\right)\right)\right\}^{\prime \prime}+\frac{b_{j} \epsilon^{3}}{3 !}\left\{\log \left(f\left(x_{j}\right)\right)\right\}^{\prime \prime \prime}+\frac{\epsilon^{4}}{4 !}\left\{\log \left(f\left(z_{j}\right)\right)\right\}^{\prime \prime \prime \prime}\right\}\right)\right\},
\end{aligned}
$$

where $E_{b_{2}, \ldots, b_{d}}$ denotes expectation with respect to $b_{2}, \ldots, b_{d}$, holding $\epsilon, b_{1}, x_{1}, x_{j}$ and $z_{j}(j=$ $2, \ldots, d)$ fixed; and for $j=2, \ldots, d, z_{j}$ lies between $x_{j}$ and $x_{j}+b_{j} \epsilon$. Since $b_{j} ; j=2, \ldots, d$ are iid, as $d \rightarrow \infty$, conditional on $\epsilon, b_{1}, x_{1}, x_{j}$ and $z_{j}(j=2, \ldots, d)$ one can apply Lyapunov's central limit theorem.

Writing $\zeta_{j}=b_{j}\left[\epsilon\left\{\log \left(f\left(x_{j}\right)\right)\right\}^{\prime}+\frac{\epsilon^{3}}{6}\left\{\log \left(f\left(x_{j}\right)\right)\right\}^{\prime \prime \prime}\right]$, we note that conditional on $\epsilon$ and $x_{j}$, $E_{b_{j}}\left(\zeta_{j}\right)=0$ and $\operatorname{Var}_{b_{j}}\left(\zeta_{j}\right)=\left[\epsilon\left\{\log \left(f\left(x_{j}\right)\right)\right\}^{\prime}+\frac{\epsilon^{3}}{6}\left\{\log \left(f\left(x_{j}\right)\right)\right\}^{\prime \prime \prime}\right]^{2}$. Viewing $b_{j} \epsilon$ as $b_{j} \epsilon^{*} \frac{\ell}{\sqrt{d}}$, where $\epsilon^{*} \sim N(0,1) I_{\left\{\epsilon^{*}>0\right\}}$, we next show that, almost surely with respect to $\pi$,

$$
\frac{\sum_{j=2}^{d} E_{b_{j}}\left(\left|\zeta_{j}\right|^{\delta}\right)}{\left\{\sqrt{\sum_{j=2}^{d} \operatorname{Var}_{b_{j}}\left(\zeta_{j}\right)}\right\}^{\delta}} \rightarrow 0
$$

for $\delta=4$.

First note that $\epsilon \equiv \epsilon^{*} \frac{\ell}{\sqrt{d}}$, where $\epsilon^{*} \sim N(0,1) I_{\left\{\epsilon^{*}>0\right\}}$, and so, for any $\zeta>0$,

$$
\sum_{d=1}^{\infty} P\left(\epsilon^{*} \frac{\ell}{\sqrt{d}}>\zeta\right)<\left(\frac{\ell}{\zeta}\right)^{4} E\left(\epsilon^{* 4}\right) \sum_{d=1}^{\infty} \frac{1}{d^{2}}<\infty .
$$

That is, $\epsilon \equiv \epsilon^{*} \frac{\ell}{\sqrt{d}} \stackrel{a . s}{\longrightarrow} 0$, a.s. denoting "almost surely". Thus, there exists a null set $\mathcal{N}_{\epsilon}$ 
(with respect to the distribution of $\epsilon^{*}$ ) such that for all $\omega_{\epsilon} \in \mathcal{N}_{\epsilon}^{c}, \epsilon \equiv \epsilon^{*}\left(\omega_{\epsilon}\right) \frac{\ell}{\sqrt{d}} \rightarrow 0$, as $d \rightarrow \infty$. Now observe that, for any given $\omega_{\epsilon} \in \mathcal{N}_{\epsilon}^{c}$, as $d \rightarrow \infty, \frac{1}{d-1} \sum_{j=2}^{d} E_{b_{j}}\left(\left|\zeta_{j}\right|^{\delta}\right)=$ $\frac{1}{d-1} \sum_{j=2}^{d}\left[\epsilon\left\{\log \left(f\left(x_{j}\right)\right)\right\}^{\prime}+\frac{\epsilon^{3}}{6}\left\{\log \left(f\left(x_{j}\right)\right)\right\}^{\prime \prime \prime}\right]^{4} \stackrel{\text { a.s. }}{\longrightarrow} E_{x_{2}}\left[\epsilon\left\{\log \left(f\left(x_{2}\right)\right)\right\}^{\prime}+\frac{\epsilon^{3}}{6}\left\{\log \left(f\left(x_{2}\right)\right)\right\}^{\prime \prime \prime}\right]^{4}$, by the strong law of large numbers (SLLN). The expectation, which is with respect to $x_{2}$, is clearly finite, due to the assumptions (13), (14), (15) of DB and the Cauchy-Schwartz inequality. In other words, given $\omega_{\epsilon} \in \mathcal{N}_{\epsilon}^{c}$, there exists a null set $\mathcal{N}_{1}$ (with respect to $f$ ) such that for all $\omega \in \mathcal{N}_{1}^{c}$, the convergence takes place deterministically. Also,

$$
\begin{aligned}
\frac{1}{d-1} \sum_{j=2}^{d} \operatorname{Var}_{b_{j}}\left(\zeta_{j}\right) & =\frac{1}{d-1} \sum_{j=2}^{d}\left[\epsilon\left\{\log \left(f\left(x_{j}\right)\right)\right\}^{\prime}+\frac{\epsilon^{3}}{6}\left\{\log \left(f\left(x_{j}\right)\right)\right\}^{\prime \prime \prime}\right]^{2} \\
& \stackrel{\text { a.s. }}{\longrightarrow} E_{x_{2}}\left[\epsilon\left\{\log \left(f\left(x_{2}\right)\right)\right\}^{\prime}+\frac{\epsilon^{3}}{6}\left\{\log \left(f\left(x_{2}\right)\right)\right\}^{\prime \prime \prime}\right]^{2},
\end{aligned}
$$

which is again finite, thanks to (13), (14) and (15) of DB, and the Cauchy-Schwartz inequality. Let $\mathcal{N}_{2}$ denote the null set (with respect to $f$ ) such that deterministic convergence takes place for all $\omega \in \mathcal{N}_{2}^{c}$. Let $\mathbb{N}_{1}=\mathcal{N}_{\epsilon} \otimes \mathcal{N}_{1} \cup \mathcal{N}_{\epsilon} \otimes \mathcal{N}_{2}$, where $\otimes$ denotes cartesian product. Then $\mathbb{N}_{1}$ is a null set with respect to the distribution of $\epsilon$ and $f$. For $\omega \in \mathbb{N}_{1}^{c}$, we have, for $\delta=4$, $\sum_{j=2}^{d} E_{b_{j}}\left(\left|\zeta_{j}\right|^{\delta}\right)=O(d)$, and $\sum_{j=2}^{d} \operatorname{Var}_{b_{j}}\left(\zeta_{j}\right)=O(d)$. Hence, for $\delta=4$, and for $\omega \in \mathbb{N}_{1}^{c}$, we have,

$$
\frac{\sum_{j=2}^{d} E_{b_{j}}\left(\left|\zeta_{j}\right|^{\delta}\right)}{\left\{\sqrt{\sum_{j=2}^{d} \operatorname{Var}_{b_{j}}\left(\zeta_{j}\right)}\right\}^{\delta}}=O\left(d^{-1}\right) \rightarrow 0
$$

Thus, Lyapunov's central limit theorem applies, and we have the following:

$$
\frac{\sum_{j=2}^{d} b_{j}\left[\epsilon\left\{\log \left(f\left(x_{j}\right)\right)\right\}^{\prime}+\frac{\epsilon^{3}}{6}\left\{\log \left(f\left(x_{j}\right)\right)\right\}^{\prime \prime \prime}\right]}{\sqrt{\sum_{j=2}^{d}\left[\epsilon\left\{\log \left(f\left(x_{j}\right)\right)\right\}^{\prime}+\frac{\epsilon^{3}}{6}\left\{\log \left(f\left(x_{j}\right)\right)\right\}^{\prime \prime \prime}\right]^{2}}} \stackrel{\mathcal{L}}{\rightarrow} N(0,1),
$$

for almost all $\epsilon$ and $x_{2}, \ldots, x_{d}$. Also note that, the square of the denominator of (6) is given by

$$
\sum_{j=2}^{d}\left[\epsilon\left\{\log \left(f\left(x_{j}\right)\right)\right\}^{\prime}+\frac{\epsilon^{3}}{6}\left\{\log \left(f\left(x_{j}\right)\right)\right\}^{\prime \prime \prime}\right]^{2}=\epsilon^{2} \sum_{j=2}^{d}\left[\left\{\log \left(f\left(x_{j}\right)\right)\right\}^{\prime}\right]^{2}+\Delta
$$

where

$$
\Delta=\frac{\epsilon^{4}}{6} \sum_{j=2}^{d} 2\left\{\log \left(f\left(x_{j}\right)\right)\right\}^{\prime}\left\{\log \left(f\left(x_{j}\right)\right)\right\}^{\prime \prime \prime}+\frac{\epsilon^{6}}{36} \sum_{j=2}^{d}\left[\left\{\log \left(f\left(x_{j}\right)\right)\right\}^{\prime \prime \prime}\right]^{2} .
$$

With the representation $\epsilon \equiv \epsilon^{*} \frac{\ell}{\sqrt{d}}$, where $\epsilon^{*} \sim N(0,1) I_{\left\{\epsilon^{*}>0\right\}}$, for $\omega_{\epsilon} \in \mathcal{N}_{\epsilon}^{c}$, the first term of (7) is given by $\epsilon^{* 2} \frac{\ell^{2}}{d} \sum_{j=2}^{d}\left[\left\{\log \left(f\left(x_{j}\right)\right)\right\}^{\prime}\right]^{2}$. As $d \rightarrow \infty$, by SLLN, there exists a null set $\mathcal{N}_{3}$ with respect to $f$ such that for all $\omega \in \mathcal{N}_{3}^{c}$,

$$
\frac{1}{d-1} \sum_{j=2}^{d}\left[\left\{\log \left(f\left(x_{j}\right)\right)\right\}^{\prime}\right]^{2}=-\frac{1}{d-1} \sum_{j=2}^{d}\left\{\log \left(f\left(x_{j}\right)\right)\right\}^{\prime \prime} \rightarrow \mathbb{I},
$$

where $\mathbb{I}$ is the information matrix corresponding to the density $f$. 
Also,

$$
\begin{aligned}
\Delta & =\frac{\epsilon^{* 4} \ell^{4}}{6 d} \frac{1}{d} \sum_{j=2}^{d} 2\left\{\log \left(f\left(x_{j}\right)\right)\right\}^{\prime}\left\{\log \left(f\left(x_{j}\right)\right)\right\}^{\prime \prime \prime}+\frac{\epsilon^{* 6} \ell^{6}}{36 d^{2}} \frac{1}{d} \sum_{j=2}^{d}\left[\left\{\log \left(f\left(x_{j}\right)\right)\right\}^{\prime \prime \prime}\right]^{2} \\
& \stackrel{a . s_{.}}{\longrightarrow} 0
\end{aligned}
$$

since

$$
\begin{gathered}
\frac{1}{d} \sum_{j=2}^{d} 2\left\{\log \left(f\left(x_{j}\right)\right)\right\}^{\prime}\left\{\log \left(f\left(x_{j}\right)\right)\right\}^{\prime \prime \prime} \stackrel{\text { a.s. }}{\longrightarrow} E_{f}\left[2\left\{\log \left(f\left(x_{2}\right)\right)\right\}^{\prime}\left\{\log \left(f\left(x_{2}\right)\right)\right\}^{\prime \prime \prime}\right]<\infty, \\
\frac{1}{d} \sum_{j=2}^{d}\left[\left\{\log \left(f\left(x_{j}\right)\right)\right\}^{\prime \prime \prime}\right]^{2} \stackrel{\text { a.s. }}{\longrightarrow} E_{f}\left[\left\{\log \left(f\left(x_{2}\right)\right)\right\}^{\prime \prime \prime}\right]^{2}<\infty,
\end{gathered}
$$

$\frac{\epsilon^{* 4}}{d} \stackrel{a . s}{\longrightarrow} 0$, and $\frac{\epsilon^{* 6}}{d^{2}} \stackrel{a . s .}{\longrightarrow} 0$. Hence, there exists a null set $\mathcal{N}_{4}$ (with respect to $f$ ) such that for all $\omega \in \mathcal{N}_{4}^{c}, \Delta(\omega) \rightarrow 0$ as $d \rightarrow \infty$.

We now deal with the last term of (4) given by $\sum_{j=2}^{d} \frac{\epsilon^{4}}{4 !}\left\{\log \left(f\left(z_{j}\right)\right)\right\}^{\prime \prime \prime \prime}$. This tends to zero almost surely as $d \rightarrow \infty$ because $\epsilon^{4} \equiv \epsilon^{* 4} \frac{\ell^{4}}{d} \stackrel{a . s}{\longrightarrow} 0$, and

$$
\frac{1}{d} \sum_{j=2}^{d}\left\{\log \left(f\left(z_{j}\right)\right)\right\}^{\prime \prime \prime \prime \prime} \stackrel{a . s .}{\longrightarrow} E_{f}\left(\left\{\log \left(f\left(z_{2}\right)\right)\right\}^{\prime \prime \prime \prime}\right)<\infty,
$$

by (16)of DB. Let $\mathcal{N}_{5}$ denote the null set associated with the above almost sure convergence.

Let $\mathbb{N}_{2}=\mathcal{N}_{\epsilon} \otimes \mathcal{N}_{3} \cup \mathcal{N}_{\epsilon} \otimes \mathcal{N}_{4} \cup \mathcal{N}_{\epsilon} \otimes \mathcal{N}_{5}$. Further, let $\mathbb{N}=\mathbb{N}_{1} \cup \mathbb{N}_{2}$. Then $\mathbb{N}$ is a null set with respect to the distribution of $\epsilon^{*}$ and $f$. Hence, given any $\omega \in \mathbb{N}^{c}$,

$$
\left|E_{b_{2}, \ldots, b_{d}}\left[\min \left\{1, \frac{\pi\left(x_{1}+b_{1} \epsilon, \ldots, x_{d}+b_{d} \epsilon\right)}{\pi\left(x_{1}, \ldots, x_{d}\right)}\right\}\right]-E_{b_{2}, \ldots, b_{d}}\left[\min \left\{1, e^{X}\right\}\right]\right| \rightarrow 0, \text { as } d \rightarrow \infty
$$

where

$$
X \sim N\left(\eta\left(x_{1}, b_{1}, \epsilon\right)-\frac{(d-1) \epsilon^{2}}{2} \mathbb{I},(d-1) \epsilon^{2} \mathbb{I}\right)
$$

with

$$
\eta\left(x_{1}, b_{1}, \epsilon\right)=\log \left(f\left(x_{1}+b_{1} \epsilon\right)\right)-\log \left(f\left(x_{1}\right)\right) .
$$

We now recall the following result (Proposition 2.4 of Roberts et al. (1997)): if $X \sim N\left(\mu, \sigma^{2}\right)$, then

$$
E\left[\min \left\{1, e^{X}\right\}\right]=\Phi\left(\frac{\mu}{\sigma}\right)+e^{\left\{\mu+\frac{\sigma^{2}}{2}\right\}} \Phi\left(-\sigma-\frac{\mu}{\sigma}\right),
$$

where $\Phi$ is the standard Gaussian cumulative distribution function (cdf). Applying this result to (4) we obtain

$$
\begin{aligned}
E_{b_{2}, \ldots, b_{d}} & {\left[\min \left\{1, \frac{\pi\left(x_{1}+b_{1} \epsilon, \ldots, x_{d}+b_{d} \epsilon\right)}{\pi\left(x_{1}, \ldots, x_{d}\right)}\right\}\right] } \\
= & \Phi\left(\frac{\eta\left(x_{1}, b_{1}, \epsilon\right)-\frac{(d-1) \epsilon^{2}}{2} \mathbb{I}}{\sqrt{(d-1) \epsilon^{2} \mathbb{I}}}\right)+e^{\eta\left(x_{1}, b_{1}, \epsilon\right)} \Phi\left(-\sqrt{(d-1) \epsilon^{2} \mathbb{I}}-\frac{\eta\left(x_{1}, b_{1}, \epsilon\right)-\frac{(d-1) \epsilon^{2}}{2} \mathbb{I}}{\sqrt{(d-1) \epsilon^{2} \mathbb{I}}}\right) \\
= & \mathbb{W}\left(b_{1}, \epsilon, x_{1}\right) .
\end{aligned}
$$


Note that using Taylor series expansion around $x_{1}$, we can write (10) as

$$
\eta\left(x_{1}, b_{1}, \epsilon\right)=b_{1} \epsilon\left[\log f\left(x_{1}\right)\right]^{\prime}+\frac{\epsilon^{2}}{2}\left[\log f\left(x_{1}\right)\right]^{\prime \prime}+b_{1} \frac{\epsilon^{3}}{3 !}\left[\log f\left(\xi_{1}\right)\right]^{\prime \prime \prime},
$$

where $\xi_{1}$ lies between $x_{1}$ and $x_{1}+b_{1} \epsilon$. Re-writing $b_{1} \epsilon$ as $\frac{\ell}{\sqrt{d}} z_{1}^{*}$, where $z_{1}^{*}$ follows a $N(0,1)$ distribution, $\eta$ and $\mathbb{W}$ can be expressed in terms of $\ell$ and $z_{1}^{*}$ as

$$
\eta\left(x_{1}, z_{1}^{*}, d\right)=\frac{\ell z_{1}^{*}}{\sqrt{d}}\left[\log f\left(x_{1}\right)\right]^{\prime}+\frac{\ell^{2} z_{1}^{* 2}}{2 ! d}\left[\log f\left(x_{1}\right)\right]^{\prime \prime}+\frac{\ell^{3} z_{1}^{* 3}}{3 ! d^{\frac{3}{2}}}\left[\log f\left(\xi_{1}\right)\right]^{\prime \prime \prime}
$$

and

$$
\mathbb{W}\left(x_{1}, z_{1}^{*}, d\right)=\Phi\left(\frac{\eta\left(x_{1}, z_{1}^{*}, d\right)-\frac{z_{1}^{* 2} \ell^{2}}{2} \mathbb{I}}{\sqrt{z_{1}^{* 2} \ell^{2} \mathbb{I}}}\right)+e^{\eta\left(x_{1}, z_{1}^{*}, d\right)} \Phi\left(\frac{-\eta\left(x_{1}, z_{1}^{*}, d\right)-\frac{z_{1}^{* 2} \ell^{2} \mathbb{I}}{2}}{\sqrt{z_{1}^{* 2} \ell^{2} \mathbb{I}}}\right) .
$$

Now we consider the Taylor series expansion around $x_{1}$ of the term

$$
\begin{aligned}
& d E_{z_{1}^{*}}\left[\left(V\left(x_{1}+\frac{z_{1}^{*} \ell}{\sqrt{d}}\right)-V\left(x_{1}\right)\right) \mathbb{W}\left(z_{1}^{*}, x_{1}, d\right)\right] \\
= & d E_{z_{1}^{*}}\left[\left\{V^{\prime}\left(x_{1}\right) \frac{z_{1}^{*} \ell}{\sqrt{d}}+\frac{1}{2} V^{\prime \prime}\left(x_{1}\right) \frac{z_{1}^{* 2} \ell^{2}}{d}+\frac{1}{6} V^{\prime \prime \prime}\left(\xi_{1}\right) \frac{z_{1}^{* 3} \ell^{3}}{d^{\frac{3}{2}}}\right\} \mathbb{W}\left(z_{1}^{*}, x_{1}, d\right)\right] .
\end{aligned}
$$

From (14) it is clear that $\mathbb{W}\left(z_{1}^{*}, x_{1}, d\right)$ is continuous but not differentiable at the point 0. So, this can not be expanded as a Taylor series around 0 . Also, note that $\mathbb{W}$ is an almost surely bounded function with respect to $d$. This follows from the fact that $\Phi$ is a bounded function and that $\eta\left(x_{1}, z_{1}^{*}, d\right) \stackrel{\text { a.s. }}{\longrightarrow} 0$ as $d \rightarrow \infty$. The latter is easily proved by showing, as in (5), that each term of $\eta\left(x_{1}, z_{1}^{*}, d\right)$ tends to zero almost surely; here we need to use the facts that $E_{f}\left[\left\{\log \left(f\left(x_{1}\right)\right)\right\}^{\prime}\right]^{4}<\infty, E_{f}\left[\left\{\log \left(f\left(x_{1}\right)\right)\right\}^{\prime \prime}\right]^{2}<\infty$ and $E_{f}\left[\left|\left\{\log \left(f\left(\xi_{1}\right)\right)\right\}^{\prime \prime \prime}\right|\right]<\infty$ which follow from assumptions (13), (14) and (15) of DB. By expanding the individual terms in the expression in (14) we obtain, for appropriate $w_{1}, w_{2}, \xi_{1}$, the following:

$$
\begin{aligned}
\Phi\left(\frac{\eta\left(x_{1}, z_{1}^{*}, d\right)-\frac{z_{1}^{* 2} \ell^{2}}{2} \mathbb{I}}{\sqrt{z_{1}^{* 2} \ell^{2} \mathbb{I}}}\right) & =\Phi\left(-\frac{\sqrt{z_{1}^{* 2} \ell^{2} \mathbb{I}}}{2}\right)+\frac{1}{\sqrt{d \mathbb{I}}}\left[\log f\left(x_{1}\right)\right]^{\prime} \phi\left(-\frac{\sqrt{z_{1}^{* 2} \ell^{2} \mathbb{I}}}{2}\right)+\frac{1}{2 d \mathbb{I}} \phi^{\prime}\left(w_{1}\right), \\
\Phi\left(\frac{-\eta\left(x_{1}, z_{1}^{*}, d\right)-\frac{z_{1}^{* 2} \ell^{2}}{2} \mathbb{I}}{\sqrt{z_{1}^{* 2} \ell^{2} \mathbb{I}}}\right) & =\Phi\left(-\frac{\sqrt{z_{1}^{* 2} \ell^{2} \mathbb{I}}}{2}\right)-\frac{1}{\sqrt{d \mathbb{I}}}\left[\log f\left(x_{1}\right)\right]^{\prime} \phi\left(-\frac{\sqrt{z_{1}^{* 2} \ell^{2} \mathbb{I}}}{2}\right)+\frac{1}{2 d \mathbb{I}} \phi^{\prime}\left(w_{2}\right), \\
e^{\eta\left(x_{1}, z_{1}^{*}, d\right)} & =1+\frac{\ell z_{1}^{*}}{\sqrt{d}}\left[\log f\left(x_{1}\right)\right]^{\prime}+\frac{\ell^{2} z_{1}^{* 2}}{2 ! d}\left[\log f\left(x_{1}\right)\right]^{\prime \prime}+\frac{\ell^{3} z_{1}^{* 3}}{3 ! d^{\frac{3}{2}}}\left[\log f\left(\xi_{1}\right)\right]^{\prime \prime \prime} .
\end{aligned}
$$

Using these expanded forms and then simplifying the expression in (15), we obtain the following form of $G_{d} V(x)$ :

$$
G_{d} V(x)=V^{\prime}\left(x_{1}\right) \frac{1}{2} \ell^{2}\left(\log f\left(x_{1}\right)\right)^{\prime} E_{z_{1}^{*}}\left[z_{1}^{* 2} \mathcal{H}\left(z_{1}^{*}\right)\right]+\frac{1}{2} V^{\prime \prime}\left(x_{1}\right) \ell^{2} E_{z_{1}^{*}}\left[z_{1}^{* 2} \mathcal{H}\left(z_{1}^{*}\right)\right]+O\left(d^{-\frac{1}{2}}\right)
$$

where 


$$
\mathcal{H}\left(z_{1}^{*}\right)=2 \Phi\left(-\frac{\left|z_{1}^{*}\right| \ell \sqrt{\mathbb{I}}}{2}\right)=2\left[1-\Phi\left(\frac{\left|z_{1}^{*}\right| \ell \sqrt{\mathbb{I}}}{2}\right)\right] .
$$

Hence, the limiting form of our generator is Langevin and is given by

$$
G V(x)=\frac{1}{2} g(\ell)\left(\log f\left(x_{1}\right)\right)^{\prime} V^{\prime}\left(x_{1}\right)+\frac{g(\ell)}{2} V^{\prime \prime}\left(x_{1}\right),
$$

where $g(\ell)$ is given by (18) of DB. Since $G_{d} V(x)$ and $V^{\prime \prime}\left(x_{1}\right)$ are bounded, and $G_{d} V(x)$ converges pointwise to $G V(x)$, Dominated Convergence Theorem implies that

$$
\lim _{d \rightarrow \infty} E\left|G_{d} V(x)-G V(x)\right| \rightarrow 0
$$

\section{S-6.2 Proof of Theorem 3.2 of DB}

Proof. We can write down the generator $G_{d} V(x)$ as follows:

$$
\begin{aligned}
G_{d} V(x)=\frac{d}{2^{d}} P\left(\chi_{1}=1\right) \int_{0}^{\infty} & \sum_{b_{1} \in\{-1,+1\}}\left[\left(V\left(x_{1}+b_{1} \epsilon\right)-V\left(x_{1}\right)\right)\right. \\
& \left.\times E\left\{\begin{array}{l}
b_{2}, b_{3}, \ldots, b_{d}, \\
\chi_{2}, \chi_{3}, \ldots, \chi_{d}
\end{array}\right\}\left(\min \left\{1, \frac{\pi\left(x_{1}+b_{1} \epsilon, \ldots, x_{d}+\chi_{d} b_{d} \epsilon\right)}{\pi\left(x_{1}, \ldots, x_{d}\right)}\right\}\right)\right] q(\epsilon) d \epsilon .
\end{aligned}
$$

Note that since $V$ is a function of $x_{1}$ only, if $\chi_{1}$ is equal to 0 , then no transition takes place and $V\left(x_{1}+\chi_{1} b_{1} \epsilon\right)-V\left(x_{1}\right)=0$, so that the value of the generator is 0 . In other words, the part of the generator associated with $P\left(\chi_{1}=0\right)$ is zero, and hence does not feature in (19).

Since $b_{j}$ and $\chi_{j}$ always occur as products, we have

$$
E^{E}\left\{\begin{array}{l}
b_{2}, b_{3}, \ldots, b_{d}, \\
\chi_{2}, \chi_{3}, \ldots, \chi_{d}
\end{array}\right\}=E_{\left\{b_{2} \chi_{2}, b_{3} \chi_{3}, \ldots, b_{d} \chi_{d}\right\}}
$$

Our approach to obtaining the diffusion limit in this problem will be similar to that in the previous problem, where all the components of $x$ are updated simultaneously at every iteration of TMCMC. Here we leave $\left(1-c_{d}\right)(d-1)$ terms unchanged at each step and sum over $c_{d} d$ many terms inside the exponential. We make a very vital assumption that $c_{d} \rightarrow c$, which forces $c_{d}(d-1)$ to go to $\infty$ as $d \rightarrow \infty$. We apply Lyapunov's central limit theorem as before (again the Lyapunov assumption holds good for $\delta=4$ ), to obtain, given any $\omega \in \mathbb{N}^{c}$,

$\left|E_{b_{2} \chi_{2}, \ldots, b_{d} \chi_{d}}\left[\min \left\{1, \frac{\pi\left(x_{1}+b_{1} \epsilon, \ldots, x_{d}+\chi_{d} b_{d} \epsilon\right)}{\pi\left(x_{1}, \ldots, x_{d}\right)}\right\}\right]-E_{b_{2} \chi_{d}, \ldots, b_{d} \chi_{d}}\left[\min \left\{1, e^{X}\right\}\right]\right| \rightarrow 0$, as $d \rightarrow \infty$,

where, using Lyapunov's theorem and the same techniques as before, we obtain

$$
X \sim N\left(\eta\left(x_{1}, b_{1}, \epsilon\right)-\frac{\left(c_{d} d-1\right) \epsilon^{2}}{2} \mathbb{I},\left(c_{d} d-1\right) \epsilon^{2} \mathbb{I}\right) .
$$


Analogously, we define $\mathbb{W}\left(x_{1}, z_{1}^{*}, c_{d}, d\right)$ as the following

$$
\mathbb{W}\left(x_{1}, z_{1}^{*}, c_{d}, d\right)=\Phi\left(\frac{\eta\left(x_{1}, z_{1}^{*}\right)-\frac{z_{1}^{* 2} \ell^{2} c_{d}}{2} \mathbb{I}}{\sqrt{z_{1}^{* 2} \ell^{2} c_{d} \mathbb{I}}}\right)+e^{\eta\left(x_{1}, z_{1}^{*}\right)} \Phi\left(\frac{-\eta\left(x_{1}, z_{1}^{*}\right)-\frac{z_{1}^{* 2} \ell^{2} c_{d}}{2} \mathbb{I}}{\sqrt{z_{1}^{* 2} \ell^{2} c_{d} \mathbb{I}}}\right) .
$$

Proceeding in the same way as in the previous case, we obtain

$$
G_{d} V(x)=V^{\prime}\left(x_{1}\right) \frac{1}{2} c_{d} \ell^{2} E_{z_{1}^{*}}\left[z_{1}^{* 2} \mathcal{H}\left(z_{1}^{*}, x_{1}, c_{d}, d\right)\right]+\frac{1}{2} V^{\prime \prime}\left(x_{1}\right) c_{d} \ell^{2} E_{z_{1}^{*}}\left[z_{1}^{* 2} \mathcal{H}\left(z_{1}^{*}, x_{1}, c_{d}, d\right)\right]+O\left(d^{-\frac{1}{2}}\right)
$$

where

$$
\mathcal{H}\left(z_{1}^{*}, x_{1}, c_{d}\right)=2 \Phi\left(-\frac{\left|z_{1}^{*}\right| \ell \sqrt{c_{d} \mathbb{I}}}{2}\right)=2\left[1-\Phi\left(\frac{\left|z_{1}^{*}\right| \ell \sqrt{c_{d} \mathbb{I}}}{2}\right)\right] .
$$

Finally, the limiting form of the generator in this case of partial updating based additive TMCMC turns out to be analogous to the previous case where all the components of $x$ are updated simultaneously at every step. This is given by

$$
G V(x)=\frac{1}{2} g_{c}(\ell)\left(\log f\left(x_{1}\right)\right)^{\prime} V^{\prime}\left(x_{1}\right)+\frac{g_{c}(\ell)}{2} V^{\prime \prime}\left(x_{1}\right),
$$

where the diffusion speed $g_{c}(\ell)$ is given by

$$
g_{c}(\ell)=4 c \ell^{2} \int_{0}^{\infty} u^{2} \Phi\left(-\frac{u \ell \sqrt{c \mathbb{I}}}{2}\right) \phi(u) d u .
$$

As before, the Dominated Convergence Theorem implies that

$$
\lim _{d \rightarrow \infty} E\left|G_{d} V(x)-G V(x)\right| \rightarrow 0
$$

\section{S-6.3 Proof of Theorem 4.1 of DB}

Proof. The generator function of the process can be written as

$$
\begin{aligned}
G_{d} V(x)=\frac{d^{\alpha}}{2^{d}} \int_{0}^{\infty} \sum_{b_{1} \in\{-1,+1\}}[ & \left(V\left(x_{1}+b_{1} \epsilon\right)-V\left(x_{1}\right)\right) \\
& \left.\times E_{b_{2}, \ldots, b_{d}}\left(\min \left\{1, \frac{\pi\left(x_{1}+b_{1} \epsilon, \ldots, x_{d}+b_{d} \epsilon\right)}{\pi\left(x_{1}, \ldots, x_{d}\right)}\right\}\right)\right] q(\epsilon) d \epsilon,
\end{aligned}
$$


where

$$
\begin{aligned}
E_{b_{2}, \ldots, b_{d}} \quad & {\left[\min \left\{1, \frac{\pi\left(x_{1}+b_{1} \epsilon, \ldots, x_{d}+b_{d} \epsilon\right)}{\pi\left(x_{1}, \ldots, x_{d}\right)}\right\}\right] } \\
=\quad & E_{b_{2}, \ldots, b_{d}}\left[\operatorname { m i n } \left\{1, \exp \left(\log \left(f\left(x_{1}+b_{1} \epsilon\right)\right)-\log \left(f\left(x_{1}\right)\right)\right.\right.\right. \\
& +\sum_{j=2}^{k}\left\{b_{j} \epsilon\left\{\log \left(f\left(\theta_{j}(d) x_{j}\right)\right)\right\}^{\prime}+\frac{\epsilon^{2}}{2 !}\left\{\log \left(f\left(\theta_{j}(d) x_{j}\right)\right)\right\}^{\prime \prime}+\frac{b_{j} \epsilon^{3}}{3 !}\left\{\log \left(f\left(\theta_{j}(d) x_{j}\right)\right)\right\}^{\prime \prime \prime}\right. \\
& \left.+\frac{\epsilon^{4}}{4 !}\left\{\log \left(f\left(\theta_{j}(d) z_{j}\right)\right)\right\}^{\prime \prime \prime \prime}\right\} \\
& +\sum_{j=k+1}^{d}\left\{b_{j} \epsilon\left\{\log \left(f\left(\theta_{j}(d) x_{j}\right)\right)\right\}^{\prime}+\frac{\epsilon^{2}}{2 !}\left\{\log \left(f\left(\theta_{j}(d) x_{j}\right)\right)\right\}^{\prime \prime}+\frac{b_{j} \epsilon^{3}}{3 !}\left\{\log \left(f\left(\theta_{j}(d) x_{j}\right)\right)\right\}^{\prime \prime \prime}\right. \\
& \left.\left.\left.\left.+\frac{\epsilon^{4}}{4 !}\left\{\log \left(f\left(\theta_{j}(d) z_{j}\right)\right)\right\}^{\prime \prime \prime \prime}\right\}\right)\right\}\right]
\end{aligned}
$$

Note that since $\epsilon$ can be represented, as before, as $\frac{\ell z_{1}^{*}}{d^{\frac{\alpha}{2}}}$ (where we assume that $\alpha>0$ ), and, due to assumptions (13), (14), (15) and (34) of DB, and because $k$ is finite, it is easy to see that the first sum in the expression in (28) goes to 0 almost surely. Then, we apply Lyapunov's central limit theorem on $b_{j}$ for $j=k+1, \ldots, d$, which deals with infinitely many random variables as $d \rightarrow \infty$, and we obtain, for every fixed $\omega \in \mathbb{N}^{c}$, where $\mathbb{N}$ is an appropriate null set as before,

$$
\frac{\sum_{j=k+1}^{d} b_{j}\left[\epsilon\left\{\log \left(f\left(\theta_{j}(d) x_{j}\right)\right)\right\}^{\prime}+\frac{\epsilon^{3}}{6}\left\{\log \left(f\left(\theta_{j}(d) x_{j}\right)\right)\right\}^{\prime \prime \prime}\right]}{\sqrt{\sum_{j=k+1}^{d}\left[\epsilon\left\{\log \left(f\left(\theta_{j}(d) x_{j}\right)\right)\right\}^{\prime}+\frac{\epsilon^{3}}{6}\left\{\log \left(f\left(\theta_{j}(d) x_{j}\right)\right)\right\}^{\prime \prime \prime}\right]^{2}}} \stackrel{\mathcal{L}}{\rightarrow} N(0,1) .
$$

The square of the denominator of (29) can be written as $\epsilon^{2} \sum_{j=k+1}^{d}\left[\left\{\log \left(f\left(\theta_{j}(d) x_{j}\right)\right)\right\}^{\prime}\right]^{2}+\Delta$, where

$$
\Delta=\frac{\epsilon^{4}}{6} \sum_{j=k+1}^{d} 2\left\{\log \left(f\left(\theta_{j}(d) x_{j}\right)\right)\right\}^{\prime}\left\{\log \left(f\left(\theta_{j}(d) x_{j}\right)\right)\right\}^{\prime \prime \prime}+\frac{\epsilon^{6}}{36} \sum_{j=k+1}^{d}\left[\left\{\log \left(f\left(\theta_{j}(d) x_{j}\right)\right)\right\}^{\prime \prime \prime}\right]^{2} .
$$

Representing $\epsilon$ as $\frac{\ell z_{1}^{*}}{d^{\frac{\alpha}{2}}}$, it can be seen as before that $\Delta \stackrel{a . s .}{\longrightarrow} 0$ as $d \rightarrow \infty$. Writing $u_{j}=\theta_{j}(d) x_{j}$, we have

$$
\begin{aligned}
\epsilon^{2} \sum_{j=k+1}^{d}\left[\left\{\log \left(f\left(\theta_{j}(d) x_{j}\right)\right)\right\}^{\prime}\right]^{2} & =\sum_{i=1}^{m} \frac{\ell^{2} z_{1}^{* 2}}{d^{\alpha}} \theta_{j}^{2}(d) r(i, d)\left\{\frac{1}{r(i, d)} \sum_{j=1}^{r(i, d)}\left(\frac{f^{\prime}\left(u_{j}\right)}{f\left(u_{j}\right)}\right)^{2}\right\} \\
& =\sum_{i=1}^{m} \frac{\ell^{2} z_{1}^{* 2} d^{\gamma_{i}} r(i, d)}{K_{k+i} d^{\alpha}} r(i, d)\left\{\frac{1}{r(i, d)} \sum_{j=1}^{r(i, d)}\left(\frac{f^{\prime}\left(u_{j}\right)}{f\left(u_{j}\right)}\right)^{2}\right\}
\end{aligned}
$$

As $d \rightarrow \infty$, almost surely,

$$
\frac{1}{r(i, d)} \sum_{j=1}^{r(i, d)}\left(\frac{f^{\prime}\left(u_{j}\right)}{f\left(u_{j}\right)}\right)^{2} \rightarrow E\left[\left\{\frac{f^{\prime}(U)}{f(U)}\right\}^{2}\right]=\mathbb{I} .
$$


Hence, as $d \rightarrow \infty$, almost surely,

$$
\epsilon^{2} \sum_{j=k+1}^{d}\left[\left\{\log \left(f\left(\theta_{j}(d) x_{j}\right)\right)\right\}^{\prime}\right]^{2} \rightarrow \ell^{2} z_{1}^{* 2} \xi^{2} \mathbb{I}
$$

where

$$
\xi^{2}=\lim _{d \rightarrow \infty} \sum_{i=1}^{m} \frac{d^{\gamma_{i}} r(i, d)}{K_{k+i} d^{\alpha}}
$$

is finite due to (34) of DB and the fact that $m$ is finite. Also, as before, the fourth order terms involved in (28) go to zero almost surely. Hence, given any $\omega \in \mathbb{N}^{c}$,

$$
\left|E_{b_{2}, \ldots, b_{d}}\left[\min \left\{1, \frac{\pi\left(x_{1}+b_{1} \epsilon, \ldots, x_{d}+b_{d} \epsilon\right)}{\pi\left(x_{1}, \ldots, x_{d}\right)}\right\}\right]-E_{b_{2}, \ldots, b_{d}}\left[\min \left\{1, e^{X}\right\}\right]\right| \rightarrow 0, \text { as } d \rightarrow \infty
$$

where

$$
X \sim N\left(\eta\left(x_{1}, b_{1}, \epsilon\right)-\frac{(d-1) \epsilon^{2}}{2} \xi^{2} \mathbb{I},(d-1) \epsilon^{2} \xi^{2} \mathbb{I}\right) .
$$

We then follow a similar approach as in the previous two cases to obtain

$$
\mathbb{W}\left(z_{1}^{*}, x_{1}, d, \xi\right)=\Phi\left(\frac{\eta\left(x_{1}, z_{1}^{*}, d\right)-\frac{z_{1}^{* 2} \ell^{2} \xi^{2}}{2} \mathbb{I}}{\sqrt{z_{1}^{* 2} \ell^{2} \xi^{2} \mathbb{I}}}\right)+e^{\eta\left(x_{1}, z_{1}^{*}\right)} \Phi\left(\frac{-\eta\left(x_{1}, z_{1}^{*}, d\right)-\frac{z_{1}^{* 2} \ell^{2} \xi^{2}}{2} \mathbb{I}}{\sqrt{z_{1}^{* 2} \ell^{2} \xi^{2} \mathbb{I}}}\right)
$$

This expression when simplified yields the following expression for the generator term:

$$
G_{d} V(x)=V^{\prime}\left(x_{1}\right) \frac{1}{2} \ell^{2} E_{z_{1}^{*}}\left[z_{1}^{* 2} \mathcal{H}\left(z_{1}^{*}, x_{1}, \xi\right)\right]+\frac{1}{2} V^{\prime \prime}\left(x_{1}\right) \ell^{2} E_{z_{1}^{*}}\left[z_{1}^{* 2} \mathcal{H}\left(z_{1}^{*}, x_{1}, \xi\right)\right]+O\left(d^{-\frac{1}{2}}\right)
$$

where

$$
\mathcal{H}\left(z_{1}^{*}, x_{1}, \xi\right)=2 \Phi\left(-\frac{\left|z_{1}^{*}\right| \ell \xi \sqrt{\mathbb{I}}}{2}\right)=2\left[1-\Phi\left(\frac{\left|z_{1}^{*}\right| \ell \xi \sqrt{\mathbb{I}}}{2}\right)\right] .
$$

By the same arguments as in the previous cases, we have

$$
\lim _{d \rightarrow \infty} E\left|G_{d} V(x)-G V(x)\right| \rightarrow 0
$$

where

$$
G V(x)=\frac{1}{2} g_{\xi}(\ell)\left(\log f\left(x_{1}\right)\right)^{\prime} V^{\prime}\left(x_{1}\right)+\frac{g_{\xi}(\ell)}{2} V^{\prime \prime}\left(x_{1}\right),
$$

with

$$
g_{\xi}(\ell)=4 \ell^{2} \int_{0}^{\infty}\left\{u^{2} \Phi\left(-\frac{u \ell \xi \sqrt{\mathbb{I}}}{2}\right)\right\} \phi(u) d u .
$$




\section{S-7 Calculations related to the dependent set-up}

\section{S-7.1 Verification of the conditions of Lyapunov's central limit theorem}

To apply Lyapunov's central limit theorem we need to show the following: with probability 1 with respect to $\pi$,

$$
\frac{\sum_{j=1}^{d} E\left(\frac{b_{j} \eta_{j}}{\sqrt{d}}\right)^{4}}{\left(\sqrt{\sum_{j=1}^{d} \frac{\eta_{j}^{2}}{d}}\right)^{4}}=\frac{\sum_{j=1}^{d} \frac{\eta_{j}^{4}}{d^{2}}}{\left(\frac{\|\eta\|^{2}}{d}\right)^{2}} \rightarrow 0, \text { as } d \rightarrow \infty .
$$

By Lemma 5.2 of Mattingly et al. (2011), $\frac{\|\eta\|^{2}}{d} \rightarrow 1 \pi$-almost surely as $d \rightarrow \infty$. This implies that the denominator of the left hand side of (40) goes to $1 \pi$-almost surely, as $d \rightarrow \infty$. Now, $\left(\frac{\|\eta\|^{2}}{d}\right)^{2}=\sum_{j=1}^{d} \frac{\eta_{j}^{4}}{d^{2}}+\sum_{i=1}^{d} \frac{\eta_{i}^{2}}{d}\left(\sum_{j \neq i} \frac{\eta_{j}^{2}}{d}\right)$. Except on a $\pi$-null set $\mathbb{N}$, where $\sum_{j=1}^{d} \frac{\eta_{j}^{2}}{d}$ need not converge to 1 , we have, for given $\zeta>0$ and $d_{0}$ depending upon $\zeta, 1-\zeta<\sum_{j \neq i} \frac{\eta_{j}^{2}}{d}<1+\zeta$ and $1-\zeta<\sum_{i=1}^{d} \frac{\eta_{i}^{2}}{d}<1+\zeta$, for $d \geq d_{0}$. Hence, for $d \geq d_{0},-\zeta^{2}-2 \zeta<\zeta^{2}-2 \zeta=(1-\zeta)^{2}-1<$ $\sum_{i=1}^{d} \frac{\eta_{i}^{2}}{d}\left(\sum_{j \neq i} \frac{\eta_{j}^{2}}{d}\right)-1<(1+\zeta)^{2}-1=\zeta^{2}+2 \zeta$, so that $\left|\sum_{i=1}^{d} \frac{\eta_{i}^{2}}{d}\left(\sum_{j \neq i} \frac{\eta_{j}^{2}}{d}\right)-1\right|<\zeta^{2}+2 \zeta$, showing that $\sum_{i=1}^{d} \frac{\eta_{i}^{2}}{d}\left(\sum_{j \neq i} \frac{\eta_{j}^{2}}{d}\right) \rightarrow 1$ on $\mathbb{N}^{c}$, the complement of $\mathbb{N}$. Since on $\mathbb{N}^{c},\left(\frac{\|\eta\|^{2}}{d}\right)^{2} \rightarrow 1$, we must have $\sum_{j=1}^{d} \frac{\eta_{j}^{4}}{d^{2}} \rightarrow 0$ on $\mathbb{N}^{c}$, showing that Lyapunov's condition (40) holds almost surely with respect to $\pi$.

Using Lyapunov's central limit theorem on $b_{j}$, and using the result that $\frac{\|\eta\|^{2}}{d} \rightarrow 1 \pi$-almost surely as $d \rightarrow \infty$, we obtain, for sufficiently large $d$,

$$
R(x, \xi) \sim A N\left(-\ell^{2} \epsilon^{2}, 2 \ell^{2} \epsilon^{2}\right),
$$

where " $A N$ " stands for "asymptotic normal".

Now, (57) of DB and the fact that for large $d, \mathbb{Q}(x, \xi) \approx R(x, \xi)$, imply

$$
\mathbb{Q}(x, \xi) \approx-\epsilon \sqrt{\frac{2 \ell^{2}}{d}}\left(\eta_{i} b_{i}+\sum_{j=1, j \neq i}^{d} \eta_{j} b_{j}\right)-\ell^{2} \epsilon^{2}
$$

so that

$$
\left[\mathbb{Q}(x, \xi) \mid b_{i}, \epsilon\right] \sim A N\left(-\ell^{2} \epsilon^{2}-\epsilon \sqrt{\frac{2 \ell^{2}}{d}} \eta_{i} b_{i}, 2 \ell^{2} \epsilon^{2}\right) .
$$

\section{S-7.2 Expected drift}

In order to obtain the diffusion approximation, we first obtain the expected drift conditions. In order to do that, we first define, as in Mattingly et al. (2011), $\mathcal{F}_{k}$ to be the sigma algebra generated by $\left\{x^{n}, \xi^{n}, \gamma^{k}, n \leq k\right\}$, and denote the conditional expectations $E\left(\cdot \mid \mathcal{F}_{k}\right)$ by $E_{k}(\cdot)$. Following Mattingly et al. (2011) we let $x^{0}=x$ and $\xi^{1}=\xi$, and set $\xi^{0}=0$ and $\gamma^{0}=0$. We then 
note that under stationarity, $E_{k}\left(x^{k+1}-x^{k}\right)=E_{0}\left(x^{1}-x\right)$, and using (51) of DB we can write

$$
\begin{aligned}
d E_{0}\left(x_{i}{ }^{1}-x_{i}\right) & =d E_{0}\left[\gamma^{1}\left(y_{i}{ }^{1}-x_{i}{ }^{1}\right)\right] \\
& =d E_{0}\left[\alpha(x, \xi) \sqrt{\frac{2 \ell^{2}}{d}}\left(\Sigma^{\frac{1}{2}} \xi\right)_{i}\right] \\
& =\frac{1}{\eta_{i}} \lambda_{i} \sqrt{2 \ell^{2} d} E_{0}\left[\min \left\{1, e^{\mathbb{Q}(x, \xi)}\right\} \xi_{i}\right] \eta_{i}
\end{aligned}
$$

where $\alpha(x, \xi)=\min \left\{1, \frac{\pi\left(y_{i}{ }^{1}\right)}{\pi\left(x_{i}\right)}\right\}$. The last step follows from (47) of DB, noting that $\xi=$ $\sum_{i=1}^{d} \xi_{i} \phi_{i}$.

Noting that $\lambda_{i} \Sigma^{-\frac{1}{2}} \phi_{i}=\phi_{i},(55)$ and self-adjointness of $\Sigma^{-1 / 2}$ yields

$$
\begin{aligned}
\lambda_{i} \eta_{i} & =\lambda_{i}\left\langle\Sigma^{-\frac{1}{2}}\left(P^{d} x\right)+\Sigma^{\frac{1}{2}} \nabla \Psi^{d}(x), \phi_{i}\right\rangle \\
& =\lambda_{i}\left\langle\Sigma^{-\frac{1}{2}}\left(P^{d} x\right)+\Sigma^{-\frac{1}{2}} \Sigma \nabla \Psi^{d}(x), \phi_{i}\right\rangle \\
& =\left\langle P^{d} x+\Sigma^{d} \nabla \Psi^{d}(x), \phi_{i}\right\rangle \\
& =\left(P^{d} x+\Sigma^{d} \nabla \Psi^{d}(x)\right)_{i} .
\end{aligned}
$$

Thus, we can write

$$
d E_{0}\left(x_{i}{ }^{1}-x_{i}\right)=\frac{1}{\eta_{i}}\left(P^{d} x+\Sigma^{d} \nabla \Psi^{d}(x)\right)_{i} \sqrt{2 \ell^{2} d} E_{0}\left[\min \left\{1, e^{\mathbb{Q}(x, \xi)}\right\} \xi_{i}\right] .
$$

Now, writing $\mu=-\ell^{2} \epsilon^{2}-\epsilon \sqrt{\frac{2 \ell^{2}}{d}} \eta_{i} b_{i}, \sigma=\sqrt{2} \ell \epsilon$, using (43) and Proposition 2.4 of Roberts et al. (1997), it follows that

$$
\begin{aligned}
\sqrt{d} E_{0}\left[\min \left\{1, e^{\mathbb{Q}(x, \xi)}\right\} \xi_{i}\right] & \\
= & \sqrt{d} E_{b_{i} \epsilon}\left[b_{i} \epsilon E_{0}\left\{\min \left\{1, e^{\mathbb{Q}(x, \xi)}\right\} \mid b_{i}, \epsilon\right\}\right] \\
\approx & \sqrt{d} E_{b_{i} \epsilon}\left[b_{i} \epsilon\left\{\Phi\left(\frac{\mu}{\sigma}\right)+e^{\mu+\frac{\sigma^{2}}{2}} \Phi\left(-\sigma-\frac{\mu}{\sigma}\right)\right\}\right] \\
= & \sqrt{d} E_{b_{i} \epsilon}\left[b _ { i } \epsilon \left\{\Phi\left(-\frac{\ell \epsilon}{\sqrt{2}}-\frac{\eta_{i} b_{i}}{\sqrt{d}}\right)\right.\right. \\
& \left.\left.+e^{-\epsilon \sqrt{\frac{\ell^{2}}{d}} \eta_{i} b_{i}} \Phi\left(-\frac{\ell \epsilon}{\sqrt{2}}+\frac{\eta_{i} b_{i}}{\sqrt{d}}\right)\right\}\right] .
\end{aligned}
$$


Using the following Taylor's series expansions

$$
\begin{aligned}
\Phi\left(-\frac{\ell \epsilon}{\sqrt{2}}-\frac{\eta_{i} b_{i}}{\sqrt{d}}\right) & =\Phi\left(-\frac{\ell \epsilon}{\sqrt{2}}\right)-\frac{\eta_{i} b_{i}}{\sqrt{d}} \phi\left(-\frac{\ell \epsilon}{\sqrt{2}}\right)+\frac{\eta_{i}^{2}}{2 d} \phi^{\prime}\left(w_{1}\right), \\
\Phi\left(-\frac{\ell \epsilon}{\sqrt{2}}+\frac{\eta_{i} b_{i}}{\sqrt{d}}\right) & =\Phi\left(-\frac{\ell \epsilon}{\sqrt{2}}\right)+\frac{\eta_{i} b_{i}}{\sqrt{d}} \phi\left(-\frac{\ell \epsilon}{\sqrt{2}}\right)+\frac{\eta_{i}^{2}}{2 d} \phi^{\prime}\left(w_{2}\right) \\
e^{-\epsilon \sqrt{\frac{2 \ell^{2}}{d}} \eta_{i} b_{i}} & =1-\epsilon \sqrt{\frac{2 \ell^{2}}{d}} \eta_{i} b_{i}+\frac{\ell^{2} \epsilon^{2} \eta_{i}^{2}}{d} e^{-w_{3}}
\end{aligned}
$$

where $w_{1}$ lies between $-\frac{\ell \epsilon}{\sqrt{2}}$ and $-\frac{\ell \epsilon}{\sqrt{2}}-\frac{\eta_{i} b_{i}}{\sqrt{d}} ; w_{2}$ lies between $-\frac{\ell \epsilon}{\sqrt{2}}$ and $-\frac{\ell \epsilon}{\sqrt{2}}+\frac{\eta_{i} b_{i}}{\sqrt{d}}$, and $w_{3}$ lies between 0 and $\epsilon \sqrt{\frac{2 \ell^{2}}{d}} \eta_{i} b_{i}$, and noting that $E_{b_{i} \epsilon}\left[b_{i} \epsilon \Phi\left(-\frac{\ell \epsilon}{\sqrt{2}}\right)\right]=0,(47)$ can be easily seen to be of the form

$$
\begin{aligned}
\sqrt{d} E_{0}\left[\min \left\{1, e^{\mathbb{Q}(x, \xi)}\right\} \xi_{i}\right] \approx & \sqrt{d} E_{b_{i} \epsilon}\left[b _ { i } \epsilon \left\{\Phi\left(-\frac{\ell \epsilon}{\sqrt{2}}-\frac{\eta_{i} b_{i}}{\sqrt{d}}\right)\right.\right. \\
& \left.\left.+e^{-\epsilon \sqrt{\frac{2 \ell^{2}}{d}} \eta_{i} b_{i}} \Phi\left(-\frac{\ell \epsilon}{\sqrt{2}}+\frac{\eta_{i} b_{i}}{\sqrt{d}}\right)\right\}\right] \\
= & -\sqrt{2 \ell^{2}} \eta_{i} \times 2 \int_{0}^{\infty} u^{2} \Phi\left(-\frac{\ell u}{\sqrt{2}}\right) \phi(u) d u+O\left(d^{-\frac{1}{2}}\right) \\
\approx & -\sqrt{\frac{\ell^{2}}{2}} \eta_{i} \beta
\end{aligned}
$$

where

$$
\beta=4 \int_{0}^{\infty} u^{2} \Phi\left(-\frac{\ell u}{\sqrt{2}}\right) \phi(u) d u .
$$

Hence, we can re-write (46) as

$$
\begin{aligned}
d E_{0}\left(x_{i}{ }^{1}-x_{i}\right) & =\frac{1}{\eta_{i}}\left(P^{d} x+\nabla \Psi^{d}(x)\right)_{i} \sqrt{2 \ell^{2} d} E_{0}\left[\min \left\{1, e^{\mathbb{Q}(x, \xi)}\right\} \xi_{i}\right] \\
& =-\ell^{2} \beta\left(P^{d} x+\nabla \Psi^{d}(x)\right)_{i} .
\end{aligned}
$$

\section{S-7.3 Expected diffusion coefficient}

Now we evaluate the expected diffusion coefficients involving the cross product terms. For $1 \leq i \neq j \leq d$, we have

$$
d E_{0}\left[\left(x_{i}{ }^{1}-x_{i}\right)\left(x_{j}{ }^{1}-x_{j}\right)\right]=d E_{0}\left[\left\{\gamma^{1}\left(y_{i}{ }^{1}-x_{i}\right)\right\}\left\{\gamma^{1}\left(y_{j}{ }^{1}-x_{j}\right)\right\}\right]
$$

Check that if $i \neq j$, then the above expectation is 0 using the fact that $b_{i} b_{j} \epsilon$ has 0 mean for $i \neq j$. However for $i=j$, using (45) again, we can reduce the above expectation to

$$
\begin{aligned}
d E_{0}\left[\left(x_{i}{ }^{1}-x_{i}\right)\left(x_{j}{ }^{1}-x_{j}\right)\right] & =d E_{0}\left[\left(x_{i}{ }^{1}-x_{i}\right)^{2}\right] \\
& =d E_{0}\left[\alpha(x, \xi)\left(y_{i}{ }^{1}-x_{i}\right)^{2}\right] \\
& =2 \ell^{2} \lambda_{i}{ }^{2} E_{0}\left[\xi_{i}{ }^{2} \min \left\{1, e^{\mathbb{Q}(x, \xi)}\right\}\right] .
\end{aligned}
$$


Using the same Taylor's series expansions (48) it is easily seen that

$$
\begin{aligned}
E_{0}\left[\xi_{i}{ }^{2} \min \left\{1, e^{\mathbb{Q}(x, \xi)}\right\}\right] & \approx 4 \int_{0}^{\infty} u^{2} \Phi\left(-\frac{\ell u}{\sqrt{2}}\right) \phi(u) d u \\
& =\beta
\end{aligned}
$$

Hence,

$$
\begin{aligned}
d E_{0}\left[\left(x_{i}{ }^{1}-x_{i}\right)\left(x_{j}{ }^{1}-x_{j}\right)\right] & =2 \ell^{2} \lambda_{i}{ }^{2} E_{0}\left[\xi_{i}{ }^{2} \min \left\{1, e^{\mathbb{Q}(x, \xi)}\right\}\right] \\
& \approx 2 \ell^{2} \lambda_{i}{ }^{2} \beta \\
& =2 \ell^{2} \beta\left\langle\phi_{i}, \Sigma \phi_{i}\right\rangle .
\end{aligned}
$$

It follows that

$$
d E_{0}\left[\left(x^{1}-x\right) \otimes\left(x^{1}-x\right)\right] \approx 2 \ell^{2} \beta \Sigma^{d} .
$$

Note that, by definition,

$$
x^{k+1}=x^{k}+E_{k}\left(x^{k+1}-x^{k}\right)+\sqrt{\frac{2 \ell^{2} \beta}{d}} \Gamma^{k+1, d},
$$

where, for $k \geq 0$,

$$
\Gamma^{k+1, d}=\sqrt{\frac{d}{2 \ell^{2} \beta}}\left(x^{k+1}-x^{k}-E_{k}\left(x^{k+1}-x^{k}\right)\right) .
$$

From (51), we have, for $d$ large enough,

$$
x^{k+1} \approx x^{k}-\frac{\ell^{2} \beta}{d} m^{d}\left(x^{k}\right)+\sqrt{\frac{2 \ell^{2} \beta}{d}} \Gamma^{k+1, d},
$$

where

$$
m^{d}(x)=P^{d} x+\Sigma^{d} \nabla \Psi^{d}(x) .
$$

From the definition of $\Gamma^{k, d}$ and (55) we have, as in Mattingly et al. (2011),

$$
E_{k}\left(\Gamma^{k+1, d}\right)=0 \quad \text { and } \quad E_{k}\left(\Gamma^{k+1, d} \otimes \Gamma^{k+1, d}\right) \approx \Sigma^{d} .
$$

Thus, for large enough $d$, (56) can be viewed as the Euler scheme for simulating the finite dimensional approximation

$$
x^{k+1} \approx x^{k}-g(\ell) m^{d}\left(x^{k}\right) \Delta t+\sqrt{2 g(\ell) \Delta t} \Gamma^{k+1, d} \text { where } \Delta t=\frac{1}{d},
$$

(with drift function $m^{d}$ and covariance operator $\Sigma^{d}$ ) of the SDE

$$
\frac{d z}{d t}=-g(\ell)(z+\Sigma \nabla \Psi(z))+\sqrt{2 g(\ell)} \frac{d W}{d t}, \quad z(0)=z^{0},
$$

where $z^{0} \sim \pi, W$ is a Brownian motion in a relevant Hilbert space with covariance operator $\Sigma$, and

$$
g(\ell)=\ell^{2} \beta,
$$

is the diffusion speed. 


\section{Bibliography}

Bedard, M. (2007). Weak Convergence of Metropolis Algorithms for Non-i.i.d. Target Distributions. The Annals of Applied Probability, 17, 1222-1244.

Dey, K. K. and Bhattacharya, S. (2015). Adaptive Transformation based Markov Chain Monte Carlo. Manuscript under preparation.

Dey, K. K. and Bhattacharya, S. (2016). A Brief Tutorial on Transformation Based Markov Chain Monte Carlo and Optimal Scaling of the Additive Transformation. Brazilian Journal of Probability and Statistics. To appear.

Mattingly, J. C., Pillai, N. S., and Stuart, A. M. (2011). Diffusion Limits of the Random Walk Metropolis Algorithm in High Dimensions. The Annals of Applied Probability, 22, 881-930.

Roberts, G., Gelman, A., and Gilks, W. (1997). Weak Convergence and Optimal Scaling of Random Walk Metropolis Algorithms. The Annals of Applied Probability, 7, 110-120.

Roberts, G. O. and Rosenthal, J. S. (2001). Optimal Scaling for Various Metropolis-Hastings Algorithms. Statistical Science, 16(4), 351-367.

Roberts, G. O. and Rosenthal, R. S. (2009). Examples of Adaptive MCMC. Journal of Computational and Graphical Statistics, 18, 349-367. 